\title{
UNIQUENESS OF TOPOLOGY FOR COMMUTATIVE SEMISIMPLE F-ALGEBRAS
}

\section{R. L. CARPENTER}

Abstract. Let $B$ be an $F$-algebra and $A$ be a commutative semisimple $F$-algebra such that the spectrum of $A$ contains no isolated points. We prove that any homomorphism of $B$ onto $A$ is necessarily continuous. Let $A$ be a commutative semisimple algebra. We prove that there is at most one topology with respect to which $A$ is an $F$-algebra.

Introduction. Let $A$ be a semisimple algebra over the complex numbers. It was shown by Gelfand [3, Satz 17] that if $A$ is commutative and has an identity, then there is at most one norm (up to equivalence) which makes $A$ into a Banach algebra. C. E. Rickart gave several extensions of this result in the paper [6]. In [4] B. E. Johnson proved that the norm is unique in any semisimple Banach algebra.

E. A. Michael $[5, \S 14]$ extended the theorem on uniqueness of topology to certain types of $F$-algebras. In particular it is proved in [5] that the topology is unique for commutative semisimple $F$ algebras in which every homomorphism of the algebra onto the complex numbers is continuous. (Whether all homomorphisms of a commutative $F$-algebra onto the complex numbers are continuous is one of the outstanding questions which remain open for $F$-algebras.) In this paper we show that if $A$ is a commutative semisimple algebra, then there is at most one topology with respect to which $A$ is an $F$ algebra. We also show that if $\phi$ is a homomorphism of an $F$-algebra $B$ onto a commutative semisimple $F$-algebra $A$ whose spectrum contains no isolated points, then $\phi$ is necessarily continuous.

Uniqueness of topology. A commutative $F$-algebra is a commutative algebra over the complex numbers which is a complete $T_{2}$ topological space with respect to a topology determined by a countable family of multiplicative seminorms $\left\{\|\cdot\|_{i}\right\}, i=1,2, \cdots$. No generality is lost if the seminorms are assumed to be increasing. That is, we may assume $\|\cdot\|_{i} \leqq\|\cdot\|_{i+1}$ for $i=1,2, \cdots$. The spectrum $M(A)$ of a commutative $F$-algebra $A$ is the space of all continuous

Presented to the Society, August 27, 1970 under the title Continuity of homomorphisms and uniqueness of topology for commutative F-algebras; received by the editors February 2, 1970.

AMS 1970 subject classifications. Primary 4650.

Key words and phrases. F-algebra, commutative, semisimple, uniqueness of topology, continuity of homomorphisms. 
homomorphisms of $A$ onto the complex numbers. $M(A)$ is given the Gelfand topology. If $A$ is a commutative $F$-algebra, then $A$ can be realized as the inverse limit of a sequence of commutative Banach algebras $A_{n}$ [5]. The spectrum $M(A)$ of $A$ is the union of the spectra $M\left(A_{n}\right)$ and each $M\left(A_{n}\right)$ is embedded continuously in $M(A)$ [1], [5]. A commutative $F$-algebra $A$ is said to be semisimple if its radical is $\{0\}$. It is shown in [5] that $A$ is semisimple if and only if for an element $x$ in $A, \phi(x)=0$ for every $\phi \in M(A)$ implies $x=0$. Throughout this paper, the symbol $C$ will denote the complex numbers.

Lemma 1. Let $A$ be a commutative algebra and $\phi_{1}, \phi_{2}, \cdots, \phi_{n}$ be distinct homomorphisms of $A$ onto $C$. There is an $x$ in $A$ such that $\phi_{i}(x)=0$ for $i=1,2, \cdots, n-1$ and $\phi_{n}(x)=1$.

Proof. Since $\phi_{i} \neq \phi_{n}$ for $i \neq n$, we can find $x_{i}$ in $A$ such that $\phi_{i}\left(x_{i}\right)=0$ and $\phi_{n}\left(x_{i}\right)=1$. Let $x=\prod_{i=1}^{n-1} x_{i}$.

The following lemma appeared in [4] for a different type of topological algebra.

Lemma 2. Let $A$ be a commutative F-algebra. Let $\phi_{1}, \phi_{2}, \cdots$ be a sequence of distinct points from $M(A)$ and let $k$ be a positive integer. Then there is a $y_{k}$ in $A$ such that $\phi_{i}\left(y_{k}\right)=0$ for $1 \leqq i<k$ and $\phi_{i}\left(y_{k}\right) \neq 0$ for $i \geqq k$.

Proof. Use Lemma 1 to find $x_{j} \in A, j=1,2, \cdots$, such that $\phi_{i}\left(x_{j}\right)$ $=0$ for $i=1,2, \cdots, j-1$ and $\phi_{j}\left(x_{j}\right)=1$. Set $y_{k}=\sum_{j=k}^{\infty} E_{j} x_{j}$ where the $E_{j}$ are defined inductively. We define $E_{k}, E_{k+1}, \cdots$ by $E_{k}=1$ and, for $j>k, E_{j}=0$ if $\phi_{j}\left(\sum_{i=k}^{j-1} E_{i} x_{i}\right) \neq 0$; and if $\phi_{j}\left(\sum_{i=k}^{j-1} E_{i} x_{i}\right)=0$ we choose $E_{j}$ such that $0<E_{j}$ and $\left\|E_{j} x_{j}\right\|_{j}<2^{-j}$. Here $\|\cdot\|_{j}$ denotes the $j$ th seminorm on $A$ and we assume the seminorms are increasing. Since $\left\|E_{j} x_{j}\right\|_{j}<2^{-i}$ and the seminorms are increasing, we have that $\sum_{j=k}^{\infty} E_{j} x_{j}$ converges to an element $y_{k}$ of $A$. It is clear from the construction that $y_{k}$ has the desired properties.

The next lemma appeared in [7]. We sketch a proof and refer the reader to [7] for the details.

Lemma 3. Let $A$ be a commutative F-algebra. $A$ can be realized as the inverse limit of a sequence of Banach algebras $A_{n}$. Let $\phi \in M(A)$. If $\phi$ is isolated in each of the spectra $M\left(A_{n}\right)$ which contains it, then $\phi$ is isolated in $M(A)$.

Proof. Use the Silov idempotent theorem to obtain an idempotent $e_{n} \in A_{n}$ such that $\phi\left(e_{n}\right)=1$ and $\psi\left(e_{n}\right)=0$ for any $\psi$ in $M\left(A_{n}\right)-\{\phi\}$. The idempotents $e_{n}$ define an idempotent $e$ in $A$ such that $\phi(e)=1$ and $\psi(e)=0$ for any $\psi$ in $M(A)-\{\phi\}$. 
THEOREM 4. Let $B$ be an $F$-algebra and $A$ be a commutative semisimple F-algebra such that $M(A)$ has no isolated points. If $\Psi$ is a homomorphism of $B$ onto $A$, then $\Psi$ is continuous.

Proof. The closed graph theorem is valid for $F$-algebras [2, p. 57]. Hence if we can show the graph of $\Psi$ is closed, then we will be finished.

Let $x_{n}$ be a sequence in $B$ such that $x_{n} \rightarrow x$ in $B$ and $\Psi\left(x_{n}\right) \rightarrow y$ in $A$. Let $S=\{\phi \in M(A): \phi \Psi$ is a continuous homomorphism of $B$ on to the complex numbers $\}$. Then for $\phi \in S,(\phi \Psi)\left(x_{n}\right) \rightarrow(\phi \Psi)(x)$ and $\phi\left(\Psi\left(x_{n}\right)\right)$ $\rightarrow \phi(y)$, hence $\phi(\Psi(x))=\phi(y)$. Therefore if we can show $S$ separates the points of $A$, then we will be able to conclude that the graph of $\Psi$ is closed. We will show that $S$ separates the points of $A$ by proving that $S$ is dense in $M(A)$.

Assume there is a point $\phi$ in $M(A)$ which is not in the closure of $S$. Let $A$ be the inverse limit of a sequence of Banach algebras $A_{n}$. Since $\phi$ is not isolated in $M(A)$, Lemma 3 implies there is an integer $n$ such that $\phi$ is not isolated in $M\left(A_{n}\right)$. Since the Gelfand topology is $T_{2}$ and $\phi$ is not in the closure of $S$, we have that $M\left(A_{n}\right)$ must contain infinitely many points which are not in $S$. Choose a sequence $\phi_{1}, \phi_{2}, \cdots$ of distinct points from $M\left(A_{n}\right)-S$.

Use Lemma 2 to obtain a sequence $y_{1}, y_{2} \ldots$ of points from $A$ such that $\phi_{i}\left(y_{k}\right)=0$ for $i<k$ and $\phi_{i}\left(y_{k}\right) \neq 0$ for $i \geqq k$. Let $z_{1}, z_{2}, \cdots$ be elements of $B$ such that $\Psi\left(z_{i}\right)=y_{i}$ for $i=1,2, \cdots$. Use induction to construct a sequence $x_{1}, x_{2}, \cdots$ in $B$ such that

(i) $\max _{1 \leqq j \leqq i}\left\|\left|z_{j} \cdots z_{i} x_{i} \|\right|_{i}<2^{-i}\right.$, and

(ii) $\left|\phi_{i} \psi\left(x_{i}\right)\right|>\left(\left|\phi_{i} \Psi \sum_{j=1}^{i-1} z_{1} \cdots z_{j} x_{j}\right|+i\right)\left|\phi_{i} \Psi\left(z_{1} \cdots z_{i}\right)\right|^{-1}$. Here $\left.\|\cdot\|\right|_{i}$ denotes the $i$ th seminorm on $B$ and the seminorms on $B$ are assumed to be increasing. It is always possible to choose $x_{i}$ satisfying (i) and (ii) since $\phi_{i} \Psi$ is not continuous at zero.

Let $x=\sum_{i=1}^{\infty} z_{1} \cdots z_{i} x_{i}$. Condition (i) and the fact that the seminorms are increasing guarantee that the sum converges.

For each positive in teger $k>1$, we have

$$
\begin{aligned}
\phi_{k} \Psi(x)= & \phi_{k} \Psi\left(\sum_{i=1}^{k-1} z_{1} \cdots z_{i} x_{i}\right)+\phi_{k} \Psi\left(z_{1} \cdots z_{k} x_{k}\right) \\
& +\phi_{k} \Psi\left(z_{1} \cdots z_{k+1} x_{k+1}\right) \\
& +\phi_{k} \Psi\left[\left(z_{1} \cdots z_{k+1}\right) \sum_{i=k+2}^{\infty} z_{k+2} \cdots z_{i} x_{i}\right] \\
= & \phi_{k} \Psi\left(\sum_{i=1}^{k-1} z_{1} \cdots z_{i} x_{i}\right)+\phi_{k} \Psi\left(z_{1} \cdots z_{k} x_{k}\right) .
\end{aligned}
$$


Therefore

$$
\left|\phi_{k} \Psi(x)\right|>\left|\phi_{k} \Psi\left(z_{1} \cdots z_{k} x_{k}\right)\right|-\left|\phi_{k} \Psi\left(\sum_{i=1}^{k-1} z_{1} \cdots z_{i} x_{i}\right)\right|>k .
$$

This is impossible since $\phi_{k} \in M\left(A_{n}\right)$ for each $k$ and therefore $\left|\phi_{k}(\Psi(x))\right|$ $\leqq\|\Psi(x)\|_{n}$ where $\|\cdot\|_{n}$ is the seminorm on $A$ corresponding to the Banach algebra $A_{n}$. This contradiction implies that $S$ is dense in $M(A)$.

Since $S$ is dense in $M(A)$ and $A$ is semisimple, $S$ must separate the points of $A$. As noted earlier, knowing that $S$ separates the points of $A$ allows us to conclude that $\Psi$ is continuous.

REMARK. If we could remove the requirement that $M(A)$ has no isolated points from the hypotheses of Theorem 4 , then we would have as a special case of Theorem 4 that every homomorphism of $B$ onto the complex numbers is continuous.

THEOREM 5. Let $A$ be a semisimple commutative F-algebra with respect to a topology $\tau$ then $\tau$ is the only topology with respect to which $A$ is an F-algebra.

Proof. Assume $A$ is an $F$-algebra with respect to a second topology $\tau_{1}$. Let $i$ be the identity map from $\left(A, \tau_{1}\right)$ to $(A, \tau)$. We will use the closed graph theorem to show that $i$ is continuous. It will then follow from the open mapping theorem that $i$ must be a homeomorphism.

Let $S=\left\{\phi \in M(A, \tau): \phi \in M\left(A, \tau_{1}\right)\right\}$. We will show that $S$ is dense in $M(A, \tau)$. If $S$ is dense in $M(A, \tau)$, then $S$ separates the points of $A$. And if $S$ separates the points of $A$, then the graph of $i$ is closed.

Let $\phi$ be a point in $M(A, \tau)$. If $\phi$ is not isolated in $M(A, \tau)$, then we can show as in the proof of Theorem 4 that $\phi$ is in the closure of $S$.

Assume that $\phi$ is isolated in $M(A, \tau)$. The Silov idempotent theorem implies there is an idempotent $e$ in $A$ such that $\phi(e)=1$ and $\psi(e)=0$ for $\psi$ in $M(A, \tau)-\{\phi\}$. We do not know that $\phi$ is continuous on $\left(A, \tau_{1}\right)$; however, there is an element $\phi^{\prime}$ of $M\left(A, \tau_{1}\right)$ such that $\phi^{\prime}(e)=1[5$, p. 25]. Let $x$ be an element of $A$, then for any $\psi$ in $M(A, \tau)$ we have $\psi(x e-\phi(x) e)=0$. Since $A$ is semisimple, we have $x e=\phi(x) e$. For any $x$ in $A, \phi^{\prime}(x)=\phi^{\prime}(x e)=\phi^{\prime}(\phi(x) e)=\phi(x)$. Hence $\phi^{\prime}=\phi$ which implies $\phi$ is continuous with respect to $\tau_{1}$. Therefore $\phi$ is in $S$.

We have shown that $S$ is dense in $M(A, \tau)$. We conclude that $i$ must be a homeomorphism.

One of the most intriguing questions which remains open for $F$ algebras is the question of whether all homomorphisms of a commutative $F$-algebra onto the complex numbers are necessarily continuous. 
It is shown in [5] that for purposes of this question it is sufficient to consider semisimple commutative $F$-algebras. We have the following corollary to Theorem 5 .

Corollary 6. Let $A$ be a commutative semisimple F-algebra and $\phi$ be a homomorphism of $A$ onto $C$. If there is an F-algebra topology for $A$ with respect to which $\phi$ is continuous, then $\phi$ is already continuous on $A$.

\section{REFERENCES}

1. R. Arens, Dense inverse limit rings, Michigan Math. J. 5 (1958), 169-182. MR $21 \# 3780$.

2. N. Dunford and J. T. Schwartz, Linear operators. I: General theory, Pure and Appl. Math., vol. 7, Interscience, New York, 1958. MR 22 \#8302.

3. I. Gelfand, Normierte Ringe, Mat. Sb. 9 (51) (1941), 3-24. MR 3, 51.

4. B. E. Johnson, Continuity of derivations on conmutative algebras, Amer. J. Math. 91 (1969), 1-10. MR 39 \#7433.

5. E. A. Michael, Locally multiplicatively-convex topological algebras, Mem. Amer. Math. Soc. No. 11 (1952). MR 14, 482.

6. C. E. Rickart, The uniqueness of norm problem in Banach algebras, Ann. of Math. (2) 51 (1950), 615-628. MR 11, 670.

7. M. Rosenfeld, Commutative F-algebras, Pacific J. Math. 16 (1966), 159-166. MR 32 \#8196.

University of Houston, Houston, Texas 77004 